\title{
Goal Directed Fluid Resuscitation: A Review of Hemodynamic, Metabolic, and Monitoring Based Goals
}

\author{
Jeffrey A. Katz $\cdot$ Christopher G. Choukalas
}

Published online: 15 March 2013

(C) Springer Science + Business Media New York (outside the USA) 2013

\begin{abstract}
Aggressive intravenous fluid (IVF) resuscitation has been a mainstay of therapy for critically ill patients with hypotension and hypoperfusion. Recent evidence has emerged suggesting that overzealous fluid resuscitation may be associated with increased morbidity and mortality. Goal directed fluid therapies aim to exploit metabolic, hemodynamic, and monitor based parameters that may predict a response to fluid administration. The goal is to improve patient outcome by identifying patients who will benefit from IVF while avoiding excessive fluid administration to those who will not benefit.
\end{abstract}

Keywords Goal directed therapy · Fluid - Resuscitation · Pulse pressure variation $\cdot$ Stroke volume variation $\cdot \mathrm{PCO}_{2}$ gap · Esophageal Doppler · Pulse contour analysis · Lactate clearance · Venous oximetry · Ultrasound .

Passive leg raise

\section{Introduction}

The assessment of intravascular fluid volume or fluid responsiveness is both important and difficult. Studies in both the operating room $(\mathrm{OR})$ and the intensive care unit (ICU) demonstrate better outcomes when careful, systematic strategies of fluid management are utilized [1-3]. A number of traditional hemodynamic measurements are

J. A. Katz $(\bowtie) \cdot$ C. G. Choukalas $(\bowtie)$

Department of Anesthesia and Perioperative Care, University

of California, San Francisco, 505 Parnassus Ave. M917,

San Francisco, CA 94143, USA

e-mail: katzj2@anesthesia.ucsf.edu

C. G. Choukalas

e-mail: choukalasc@anesthesia.ucsf.edu purported to serve as targets for adequate resuscitation, and new measures and monitors proliferate. Evidence for traditional goals such as blood pressure and central venous pressure (CVP) is poor $[4,5 \cdot]$, whereas a number of newer technologies are supported by promising early studies. The concept of goal-directed fluid therapy administration has been advocated, but with little agreement on which goals are most critical. The following will be a discussion of relevant goals for fluid administration in OR and ICU patients with an emphasis on hemodynamic parameters (e.g. blood pressure, CVP, dynamic respiratory indices, and stroke volume variation), metabolic parameters (e.g., lactate clearance, venous oximetry, and $\mathrm{PCO}_{2}$ gap), and monitoring systems (e.g., vigileo, PiCCO, LiDCO, esophageal Doppler, and cardiac ultrasonography).

Importance of Fluid Management in the ICU

Sepsis and acute lung injury (ALI) or the acute respiratory distress syndrome (ARDS) is common in the ICU. Management of fluid status in patients with these disorders requires careful consideration. In the case of sepsis, aggressive fluid administration is recommended $[1,6,7]$. In the Rivers' trial, patients with severe sepsis or septic shock were randomized to receive either $6 \mathrm{~h}$ of early goal-directed fluid therapy or standard therapy. The treatment group received more fluid and achieved a higher CVP than the standard therapy group, and ultimately had a better outcome. The average CVP of patients in the treatment group in the Rivers' study was $13.8 \mathrm{~cm} \mathrm{H}_{2} \mathrm{O}$ versus $11.8 \mathrm{~cm} \mathrm{H}_{2} \mathrm{O}$ in the control group. More recent data from Boyd et al. [8••], however, suggest that a CVP greater than $12 \mathrm{~cm} \mathrm{H}_{2} \mathrm{O}$ is associated with increased mortality in sepsis. Taken together, these well-regarded analyses give conflicting recommendations for the fluid goals for patients with sepsis. 
Sepsis is a common cause of ARDS, and approaches to fluid management for these processes are at crossed purposes. In the case of ALI/ARDS, a fluid restrictive approach, whereby patients remained net fluid "even" over 7 days, was associated with improved outcomes [2]. A retrospective study [9] examined fluid administration in the early and late phases of sepsis complicated by ALI. They determined whether patients had adequate initial resuscitation, conservative late fluid treatment, both, or neither, and found that those patients who had both early aggressive resuscitation and late conservative fluid management did better than those with either strategy alone or neither.

The concept of fluid restriction has continued to gain traction outside of ALI/ARDS patients. In fact, a more positive fluid balance after surgery or critical illness has been increasingly recognized as a risk factor for postoperative complications and worsened organ system dysfunction [10-12]. Given this complexity and the demonstrated benefit of strategic fluid administration, a goaloriented approach might improve outcomes. One approach would be to pair goals for hemodynamic variables with those for metabolic variables in a stepwise fashion, in order to ensure optimal oxygen delivery without unnecessary fluid administration.

\section{Hemodynamic Parameters}

Adequate circulating volume is a necessary condition of adequate stroke volume and oxygen delivery, although assessing this volume is difficult. Static measures such as the mean arterial pressure (MAP) and CVP have tremendous face validity, a historical track record, and are easy to measure and understand. Still, these measures have their faults. As a goal for resuscitation, blood pressure seems an intuitive choice and, in fact, is a central tenet of fluid resuscitation algorithms [1, 6]. In a thought-provoking review, Takala [4] questioned the validity of MAP as a goal, given that in a number of large, well-designed trials of resuscitation, the achieved MAP dramatically exceeded the goal MAP of the proposed algorithms. The author pointed out the lack of randomized data supporting any given MAP and the fact that, regardless of MAP goal, clinicians, even in highly structured research protocols, seem to prefer a higher MAP in septic patients. The author posited that these higher MAPs are attained with escalating doses of vasoconstrictors, a practice that may, paradoxically, reduce end organ perfusion. In addition, even a seemingly adequate MAP can fail to protect from organ ischemia [13]. As a goal for fluid resuscitation, the MAP is probably necessary, but not sufficient.

The case for the CVP as a measure of circulating volume or fluid responsiveness is similarly weak. The physiologic rationale for right atrial pressure measurement to make inferences regarding left ventricular (LV) performance is subject to a number of assumptions that are difficult to verify (e.g., consistency of biventricular compliance and pulmonary vascular resistance, and the function of the mitral, pulmonic, and tricuspid valves). Furthermore, a recent review and meta-analysis suggested that the CVP was no better than chance at predicting fluid responsiveness and that there is no CVP low or high enough to predict intravascular volume depletion or fluid overload, respectively [5]. As such, when used as a goal for fluid resuscitation, the CVP may be just as likely as not to lead to erroneous fluid administration.

By reducing venous return and increasing right ventricular afterload, positive pressure ventilation has predictable effects on stroke volume. These effects are more pronounced in patients on the steeper part of the Starling curve, where there are larger increases in stroke volume for a given increase in ventricular end-diastolic volume (e.g., a fluid bolus). The relationship between these respirophasic changes in stroke volume and position on the Starling curve can be exploited to make inferences about a patient's likely response to fluid administration. A number of metrics to approximate these changes in stroke volume [e.g., stroke volume variation (SVV), systolic pressure variation (SPV), and pulse pressure variation (PVV)] have been identified and studied. The majority utilize analyses of arterial or plethysmogram waveforms. The degree of variation in the parameter of interest is measured, often by automated software as part of integrated monitoring systems, and displayed as a percentage. For example, a respirophasic decrease in systolic blood pressure from 120 to 100 would represent a SPV of $16.6 \%$. Thresholds indicating abnormal variation vary by device, but are generally in the range of $10-15 \%$. The higher the degree of variation, the more stroke volume is changing with respiration, and, ultimately, the more likely the patient is to experience an increase in stroke volume with fluid administration.

Systolic and pulse pressure variation are perhaps the most commonly studied, and the plethysmogram variability index is among the most commonly employed. Small studies in patients undergoing abdominal surgery have shown improvement in surrogate markers such as lactate and reductions in postoperative complications, even though patients who had their hemodynamics guided by one of the respirophasic indices did not consistently receive more or less fluid than controls [14, 15••].

Perhaps the most comprehensive evaluation of this technology is a review and meta-analysis of studies employing a variety of dynamic indices (e.g., SPV, PPV, SVV, and CVP) in the assessment of volume responsiveness [16••]. Twenty-nine studies (nine in ICU patients, 20 in OR patients) enrolling 685 patients were reviewed. Areas under the receiver operating characteristic (ROC) curves for SPV, PPV, 
SVV, and CVP were $0.86,0.94,0.84$, and 0.55 , respectively, suggesting excellent discrimination for the dynamic indices, but no better than chance discrimination for CVP.

It should be noted that the use of these metrics requires that patients be intubated and on positive pressure ventilation; spontaneous or non-invasive ventilation is associated with a different set of hemodynamic effects and their relationship to volume responsiveness has not been studied. Furthermore, the majority of data were collected in patients receiving tidal volumes of at least $8 \mathrm{~mL} / \mathrm{kg}$, which is higher than recommended for patients with ALI and ARDS. Patients also need to be in a sinus rhythm. Atrial fibrillation and frequent ectopy will alter the variation in arterial waveform amplitude independent of respirophasic changes, thereby obscuring interpretation and trend towards over estimation of variability. Finally, because such analyses require patients be synchronous with mechanical ventilation, study patients were generally deeply sedated, if not paralyzed. These pitfalls may limit generalizability of findings to ICU patients, who are often not sedated as heavily. Another important pitfall to dynamic respirophasic indices is that these measurements do not predict fluid responsiveness in patients with an open chest [17], which can occur after complex cardiac surgery [18].

This lack of generalizability to a large subset of critically ill patients remains the biggest fallback of the dynamic indices, and the assessment of volume responsiveness in patients who do not fit these stringent criteria remains a significant challenge for intensivists. In particular, the assessment of volume responsiveness in nonintubated patients or patients breathing on a spontaneous ventilator mode is a topic that has received much attention. Soubrier and colleagues investigated PPV and SPV in spontaneously breathing patients using normal tidal volumes versus forced inspiratory and expiratory efforts to simulate respirophasic changes seen during mechanical ventilation [19]. Interestingly, the forced inspiratory and expiratory breaths diminished the predictive value of PPV in spontaneously breathing patients. During normal tidal volumes, the ROC curve for PPV and SPV were 0.81 and 0.82 , respectively. Certainly, these compare favorably to CVP but perform worse than PPV under ideal circumstances. Their cut off point of $12 \%$ PPV had a high specificity $(92 \%)$ but low sensitivity $(63 \%)$. Not surprisingly, the larger percentage PPV, the more likely the patient was volume responsive [19] with spontaneous breathing. It must be stated that this was a small prospective study and the results have not been reproduced. The use of pulse pressure variation and systolic pressure variation in spontaneously breathing patients continues to be an active area of clinical research and, with time, there may be more data to support the use of PPV in spontaneously breathing patients.
Currently, the only validated marker for fluid responsiveness in spontaneously breathing patients (whether on a spontaneous ventilator mode or non-intubated) is the response to a passive leg raise (PLR). A PLR maneuver mimics a rapid fluid bolus by displacing pooled venous blood in the lower extremities back into the patient's central circulation. Volume responsive patients will have an improvement in MAP or stroke volume variation. In a radio-nucleotide study from the 1980s, a PLR produced the equivalent of a 150-200 mL fluid bolus for each leg raised [20]. Furthermore, the passive leg raise maneuver is an effective tool in patients who are on a spontaneous ventilator mode or who have atrial based arrhythmias, because the outcome of interest, MAP, is not dependent on variation. The sensitivity and specificity of passive leg raise is 81 and $93 \%$, respectively, for predicting fluid responsiveness [21]. The only significant contraindication to PLR is abdominal compartment syndrome because the blood is unable to return to the central circulation secondary to the high abdominal pressures. Preau and colleagues studied non-intubated patients with severe sepsis and pancreatitis and used the PLR in conjunction with stroke volume variation, pulse pressure variation, and peak flow velocity from a femoral arterial line [22•*]. They showed that a change in pulse pressure of $9 \%$ was non-inferior to an echocardiographic change in stroke volume of $10 \%$ with a passive leg raise in predicting fluid responsiveness. They also showed that femoral artery peak systolic velocity (measured with M-Mode bedside ultrasound) change of $8 \%$ was as accurate as the standard (PPV, SPV) parameters. The patients with "positive" PLR maneuvers had high sensitivity and specificity for volume responsiveness.

There are a multitude of hemodynamic parameters that can be used to help direct fluid therapy-PPV, SPV, SVV, PLR. Each parameter has its associated area under the curve ROC that determine its efficacy, and, while some may be more efficacious than others, each parameter has at least one major pitfall that limits its generalized use in the ICU. It is essential that intensivists understand how to use each tool to manage fluid therapy during the continuum of intensive care (i.e., mechanical ventilation to extubation) for an individual patient as well as the broad range of patients that are cared for in the ICU.

\section{Metabolic Parameters}

Although an adequate perfusion pressure is often a sign of appropriate resuscitation, occult hypoperfusion is possible in the setting of seemingly adequate hemodynamic conditions [13]. Once goals for an adequate circulation are met, metabolic goals can be addressed to detect and treat such hypoperfusion. Lactate clearance and venous oximetry are well-validated metabolic goals of fluid resuscitation. 
Table 1 Comparison of trials of lactate clearance in sepsis

\begin{tabular}{lll}
\hline & Jones et al. [23] & Jansen et al. [24] \\
\hline Protocol duration & $6 \mathrm{~h}$ & $8 \mathrm{~h}$ \\
Monitoring interval & Every $2 \mathrm{~h}$ & Every $2 \mathrm{~h}$ \\
Goal & $10 \%$ lactate clearance & $20 \%$ lactate clearance \\
Fluid totals (L) & Control: 4.3 & Control: 2.2 \\
& Intervention: $4.5^{\mathrm{ns}}$ & Intervention: $2.7 *$ \\
Outcomes & Non-inferiority with regard to mortality & Lower mortality in multivariate analysis. \\
& & Less time on ventilator, in ICU \\
\hline
\end{tabular}

$n s$ not significant

$* p<0.05$ oxygen consumption $\left(\mathrm{VO}_{2}\right)$. Patients with low $\mathrm{ScvO}_{2}$ that have been normalized after goal directed fluid therapy may still be at risk for complications from micro-circulatory insufficiency [28]. A proposed marker for this ongoing tissue hypoperfusion after the normalization of the central venous oxygen saturation is the $\mathrm{PCO}_{2}$ gap or the central venous to arterial carbon dioxide difference or $\mathrm{P}(\mathrm{cv}-\mathrm{a}) \mathrm{CO}_{2}$. The $\mathrm{PCO}_{2}$ gap likely represents a state of insufficient venous blood flow relative to $\mathrm{CO}_{2}$ production. In other words, it is an indicator of inadequate venous blood flow to remove carbon dioxide from tissues. In patients with septic shock, a $\mathrm{PCO}_{2}$ gap greater than $6 \mathrm{mmHg}$ may still require resuscitation despite normalized $\mathrm{ScvO}_{2}$ [29]. In a small study of high-risk surgical patients, three or more $\mathrm{PCO}_{2}$ gaps greater than $6 \mathrm{mmHg}$ was associated with a $20 \%$ postoperative complication rate [30]. The $\mathrm{PCO}_{2}$ gap, currently, is more of a marker of tissue hypoperfusion than a metabolic goal for directed therapy. It is an important marker to identify those who still remain underresuscitated despite normalization of $\mathrm{Scv}_{2}$. Further studies are required to assess if correcting the $\mathrm{PCO}_{2}$ gap as a goal in directed therapy will improve patient outcomes.

\section{Monitoring Systems}

The evaluation of fluid responsiveness and goal directed therapy has sparked development of invasive patient monitors that utilize pulse contour analysis and transpulmonary thermodilution as well as renewed interest in non-invasive ultrasound based monitors (esophageal Doppler, critical care ultrasonography) and new technologies, such as endotracheal tube- and pulse wave transit time-based stroke volume estimators. These devices aim to address hemodynamic, rather than metabolic goals, to help determine fluid responsiveness. The pulse contour analysis devices such as the Vigileo (Edwards Life Science, Irvine, CA), PiCCO (Philips, Andover, MA), and LiDCO (LiDCO Ltd, Cambridge, United Kingdom) use the arterial waveform to derive stroke volume. In Marik's review of the dynamic indices, SVV compared favorably to PPV and SPV with ROC estimated area-under-the-curve of $84 \%$ and sensitivity and specificity of 82 and $86 \%$, respectively [16॰*]. Each device

Central venous and mixed venous oxygenation saturation reflect the relationship of oxygen delivery $\left(\mathrm{DO}_{2}\right)$ and 
Table 2 Summary of hemodynamic monitors

\begin{tabular}{llll}
\hline Monitor & Type of technology & Limitations & Evidence \\
\hline Vigileo & Pulse contour analysis & No internal calibration & RCT: decreased serum lactate and post op complications \\
& & & {$[15,33]$} \\
LiDCO & $\begin{array}{l}\text { Pulse contour analysis internal } \\
\text { calibration with lithium }\end{array}$ & $\begin{array}{c}\text { Requires placement of proprietary } \\
\text { arterial line (axillary, brachial, }\end{array}$ & $\begin{array}{c}\text { RCT: major abdominal surgery and prolonged surgery } \\
\text { patients had decreased post-op complications by 52 } \%\end{array}$ \\
& thermodilution & femoral) & {$[32 \bullet, 33]$} \\
PiCCO & $\begin{array}{l}\text { Pulse contour analysis internal } \\
\text { calibration with transpulmonary }\end{array}$ & $\begin{array}{c}\text { Requires placement of proprietary } \\
\text { thermodilution }\end{array}$ & RCT: off pump CABG showing improved intra-operative \\
& hemodynamics and decreased post operative infections
\end{tabular}

$R C T$ randomized, controlled trial, $C A B G$ coronary artery bypass grafting

attempts to better enable the intensivist to provide goal directed therapy by continuously displaying SVV, CO, and SVR.

Each device has been shown to improve various patient outcomes [15, 31, 32••, 33, 34••, 35]. However, each device has significant limitations including the need to purchase proprietary monitors, and the LiDCO and PiCCO systems require the placement of a proprietary arterial catheter (brachial, axillary, or femoral placement). This greatly limits their utility in patients who are not considered high risk. Unlike the Vigileo, these two systems allow for internal calibration of the pulse contour analysis with cardiac output calculation via transpulmonary thermodilution and lithium dilution techniques, respectively. The benefit of the Vigileo system is that the monitor attaches to a standard arterial catheter and can be used at the radial artery. It does not internally validate the pulse contour analysis with a cardiac output calculation. The ease of use of the Vigileo system makes it an attractive monitoring option for goal directed therapy in critically ill patients, but accuracy of all the devices during rapid hemodynamic changes remains a major concern. Table 2 summarizes differences between these monitoring systems.

Because the esophagus and descending thoracic aorta are in close proximity, esophageal Dopplers can take advantage of this anatomic relationship to measure blood flow in the descending aorta. Cardiac output is estimated using the velocity of blood flow and an aortic cross sectional area estimate. Stroke volume and stroke volume variation can then be obtained to help assess fluid responsiveness. Many assumptions pertaining to the cross sectional area of the aorta and the distribution of flow between the aortic arch branches and the descending aorta are based on healthy volunteers [36]. These relationships may change in patients with co-morbidities and under conditions of hemodynamic instability. Esophageal Dopplers, nonetheless, are easy to use, easy to place, and provide another non-invasive option to help optimize fluid balance in mechanically ventilated patients [37]. When combined with a passive leg raise, esophageal Doppler is highly sensitive and specific for fluid responsiveness (97 and $94 \%$, respectively) [38].
Bedside ultrasonography has also been receiving much attention for its application in critically ill patients for resuscitation. It has been shown that the skill acquisition is easily accomplished, and therapeutic decisions can be made based on the interpretation of the images [39]. Bedside cardiac ultrasound assists in volume status assessment using two main views. The easiest and most reliable image is the inferior vena cava (IVC) in the subcostal view. Collapsibility of the IVC during respirophasic changes is highly suggestive of volume responsiveness whereas a congested IVC is often suggestive of circulatory overload or obstructive cardiogenic shock [40]. The second assessment of volume status is the parasternal short axis view at the mid papillary muscles where collapse of the left ventricular cavity without dilation of the right ventricle is diagnostic of hypovolemia. The finding of a full left ventricle does not rule out volume responsiveness [41]. It is also possible to use cardiac ultrasonography to measure stoke volume and stroke volume variation [42] but this requires significantly more skill in image acquisition.

Two new technologies are also being developed to guide fluid therapy. Endotracheal tube-based cardiac output monitors exploit the relationship between the electrical current generated by the flow of electrolytes through the ascending aorta and stroke volume. Pulse-wave transit time systems approximate stroke volume by calculating the time between the end of the QRS complex on electrocardiogram and the beginning of the arterial pulse on an arterial line or plethysmogram. Both have been successfully validated against thermodilution cardiac output estimates from pulmonary artery catheters [43, 44], but neither has been studied in a way that measures clinical outcomes.

\section{Summary and Conclusions}

Fluid administration is a mainstay of therapy in the ICU. Shock and inadequate volume resuscitation carry a high mortality. Common conditions such as sepsis and ARDS present challenges and competing priorities with regard to fluid balance and therapy. Little consensus exists regarding 
optimal resuscitation strategies, and the level of evidence of current literature does not support the superiority of one strategy, technology, or parameter over others. Still, administration of fluid in a goal-directed manner is associated with improved outcomes. Traditional parameters, such as the MAP and CVP, are not based on strong foundations of evidence, even though they play an important role in recommended therapies. Dynamic respiratory indices, such as the PPV, show promise in the identification of patients whose stroke volume will respond to additional fluid. New monitoring systems have been developed to help tailor goal directed therapy to individual patients. Beyond the hemodynamic parameters, resuscitating to metabolic goals such as a target $\mathrm{ScvO}_{2}$ or lactate clearance has been shown to reduce mortality in septic and cardiogenic shock, suggesting such goals may be worth achieving.

Disclosure Jeffrey A. Katz and Christopher G. Choukalas have no conflict of interest.

\section{References}

Papers of particular interest, published recently, have been highlighted as:

- Of importance

- Of major importance

1. Rivers E, Nguyen B, Havstad S, et al. Early goal-directed therapy in the treatment of severe sepsis and septic shock. N Engl J Med. 2001;345:1268-77.

2. ARDS Network. Comparison of two fluid-management strategies in acute lung injury. N Engl J Med. 2006;354(24):2564-75.

3. Hamilton MA, Cecconi M, Rhodes, A. A systematic review and meta-analysis on the use of preemptive hemodynamic intervention to improve postoperative outcomes in moderate and high-risk surgical patients. Anesth Analg. 2010, Octover, electronic publication ahead of print.

4. Takala J. Should we target blood pressure in sepsis? Crit Care Med. 2010;37(10):1-7.

5. - Marik, PE, Baram, M, Vahid, B. Does central venous pressure predict fluid responsiveness? Chest. 2008;134(1):172-7. This meta-analysis describes the poor relationship between CVP and total body volume and, more importantly, volume responsiveness. Critics argue effect size heterogeneity invalidates the results.

6. Dellinger RP, Levy MM, Carlet JM, et al. Surviving Sepsis Campaign: international guidelines for management of severe sepsis and septic shock. Intensive Care Med. 2008;34:17-60.

7. Russell JA. Management of Sepsis. New Engl J Med. 2006; 355(16):1699-713.

8. • Boyd, JH, Forbes, J, Nakada, TA, Walley, KR, Russell, JA. Fluid resuscitation in septic shock: a positive fluid balance and elevated central venous pressure are associated with increased mortality. Crit Care Med. 2011;39(2):259-65. This paper essentially outlines that total body hypervolemia is associated with worse patient outcomes. The significance of goal directed fluid therapy is to give intravenous fluid when appropriate and necessary and avoid when not.
9. Murphy CV, Schramm GE, Doherty JA, et al. The importance of fluid management in acute lung injury secondary to septic shock. Chest. 2009;136(1):102-9.

10. Rahbari NN, Zimmermann JB, Schmidt T, Koch M, Weigand MA, Weitz J. Meta-analysis of standard, restrictive and supplemental fluid administration in colorectal surgery. Br J Surg. 2009;96:331-41.

11. Danish Study Group on Perioperative Fluid Therapy. Effects of intravenous fluid restriction on postoperative complications: comparison of two perioperative fluid regimens: a randomized assessor-blinded multicenter trial. Ann Surg. 2003;238:641-8.

12. Prowle JR, Horng-Ruey C, Bagshaw SM, Bellomo R. Clinical review: volume of fluid resuscitation and the incidence of acute kidney injury- a systematic review. Crit Care. 2012;16:230-5.

13. Abuelo JG. Normotensive ischemic acute renal failure. New Engl J Med. 2007;357(8):797-805.

14. Forget P, Lois F, DeKock M. Goal-directed fluid management based on the pulse oximeter-derived pleth variability index reduces lactate levels and improves fluid management. Anesth Analg. 2010;111(4):910-4.

15. •• Benes, J, Chytra, I, Altmann, P, Hluchy, M, Kasal, E, Svitak, R, Pradl, R, Stepan, M. Intraoperative fluid optimization using stroke volume variation in high risk surgical patients: results of prospective randomized study. Crit Care. 2010;14:118. Randomized control trial in abdominal surgery patients using Vigileo showing decreased post op complications and length of stay but no mortality difference.

16. $\bullet$ Marik, PE, Cavallazzi, R, Vasu, T. Dynamic changes in arterial waveform-derived variables and fluid responsiveness: A systematic review of the literature. Crit Care Med. 2009;37(9):2642-7. A classic paper and review of the literature regarding arterial waveform-derived variables for fluid responsiveness.

17. De Waal E, Rex S, et al. Dynamic preload indicator fail to predict fluid responsiveness in open chest conditions. Crit Care Med. 2009;37:510-5.

18. Boeken U, Assmann A, Mehdiani A, Akhyari P, Lichtenberg A. Open chest management after cardiac operations: outcome and timing of delayed sternal closure. Eur J Cardiothorac Surg. 2011;40(5):1146-50.

19. Soubrier S, Sualnier F, et al. Can dynamic indicators help the prediction of fluid responsiveness in spontaneously breathing critically ill patients? Intensive Care Med. 2007;33:1117-24.

20. Rutlen DL, Wackers FJ, et al. Radionuclide assessment of peripheral intravascular capacity: a technique to measure intravascular volume changes in the capacitance circulation in man. Circulation. 1981;64:146-52.

21. Thiel S, Kollef M, Isakow W. Non-invasive stroke volume measurement and passive leg raising predict volume responsiveness in medical ICU patients: an observational cohort study. Crit Care. 2009;13:R111.

22. $\bullet$ Preau S, Saulnier F, et al. Passive leg raising is predictive of fluid responsiveness in spontaneously breathing patients with severe sepsis or acute pancreatitis. Crit Care Med. 2010,38:819-25. The paper shows that passive leg raising is a safe and effective measure to determine if patients are fluid responsive. If contraindications to arterial waveform based analysis is present, the results of this paper suggest that PLR should be used.

23. • Jones, AE, Shapiro, NI, Trzeciak, S, et al. Lactate clearance vs central venous oxygen saturation as goals of early sepsis therapy: A randomized clinical trial. JAMA 2010;303(8):739-46. This paper has high clinical utility as it shows that lactate clearance is non-inferior to $\mathrm{Scv} 02$ monitoring.

24. Jansen TC, Van Bommel J, Schoonderbeek FJ, et al. Early lactate-guided therapy in intensive care unit patients: a multicenter, open-label, randomized, controlled trial. Am J Resp Crit Care Med. 2010;182(6):752-61. 
25. Reinhart K, Bloos F. The value of venous oximetry. Curr Opin Crit Care. 2005;11(3):259-63.

26. Ho KM, Harding R, Chamberlain J, Bulsara M. A comparison of central and mixed venous oxygen saturation in circulatory failure. J Cardiothorac Vasc Anesth. 2010;24(3):434-9.

27. Chawla LS, Zia H, Guttierrez G, et al. Lack of equivalence between central and mixed venous oxygen saturation. Chest. 2004;126(6):1891-6.

28. Vallet B, Teboul J, Cain S, Curtis S. Venoarterial CO(2) difference during regional ischemic or hypoxic hypoxia. J Appl Physiol. 2000;89:1317-21.

29. Vallee F, Vallet B, Mathe O, et al. Central venous to arterial carbon dioxide difference: an additional target for goal directed therapy in septic shock. Intensive Care Med. 2008;34(12):22118-25.

30. Futier E, Robin E, Jabaudon M, et al. Central venous oxygen saturation and venous to arterial carbon dioxide difference as complimentary tools for goal directed therapy during high risk surgery. Crit Care. 2010;14:R193.

31. Pearse R, Dawson D, Fawcett J, et al. Early goal directed therapy after major surgery reduces complications and duration of hospital stay. A randomized controlled trial. Crit Care. 2005;9:R687-93.

32. • Lobo S, Ronchi L, Oliveria N, et al. Restrictive strategy of intraoperative fluid maintenance during optimization of oxygen delivery decreased major complications after high-risk surgery. Crit Care 2011;15:R226. Study using LiDCO system to as guide to goal directed therapy. Randomized control trial showing decreasing post-operative complications.

33. Delfino L, Giglio M, Puntillo F, et al. Hemodynamic goal directed therapy and postoperative infections: earlier is better. A systematic review and meta-analysis. Crit Care. 2011;15:R154.

34. • Hamilton M, Cecconi M, Rhodes A. A systematic review and meta-analysis of preemptive hemodynamic interventions to improve postoperative outcomes in moderate and high-risk surgical patients. Anesth Analg. 2011;112:1392-402. This meta-analysis evaluates the use of goal directed therapy in high risk (ASA 3-4) and showed decreased morbidity.

35. Smetkin A, Kirov M, Kuzkov V, et al. Single transpulmonary thermodilution and continuous monitoring of central venous oxygen saturation during off pump coronary surgery. Acta Anaesthesiol Scand. 2009;53(4):505-14.

36. Alhashemi J, Cecconi M, Hofer C. Cardiac Output Monitoring: an integrative perspective. Crit Care. 2011;15:214.

37. Singer M, Bennett D. Noninvasive optimization of left ventricular filling using esophageal Doppler. Crit Care Med. 1991;19:1132.

38. Monnet X, Rienzo M, Osman D, et al. Passive leg raise predicts fluid responsiveness in the critically ill. Crit Care Med. 2006;34(5):1402-7.

39. Manasia A, Nagarag H, et al. Feasibility and potential clinical utility of goal directed transthoracic echocardiography performed by noncardiologist intensivist using hand carried device (SonoHeart) in the critically ill. J Cardiothorac Vasc Anesth. 2005;19(2):155-9.

40. Au S, Vieillard-Baron A. Bedside echocardiography in critically ill patients: a true hemodynamic monitoring tool. J Clin Monit Comput. 2012;26:355-60.

41. Salem R, Valle F, Rusca M, Mebazaa A. Hemodynamic monitoring by echocardiography in the ICU: the role of the new echo techniques. Curr Opin Crit Care. 2008;14:561-8.

42. Charron C, Caille V, Jardin F, et al. Echocardiographic measurement of fluid responsiveness. Curr Opin Crit Care. 2006;12:249-54.

43. Wallace AW, Salahieh A, Lawrence A, Spector K, Owens C, Alonso D. Endotracheal cardiac output monitor. Anesthesiology. 2000;92(1):178-89.

44. Yamada T, Tsutsui M, Sugo Y, Sato T, Akazawa T, Sato N, Yamashita K, Ishihara H, Takeda J. Multicenter study verifying a method of noninvasive cardiac output measurement using pulse wave transit time: a comparison with intermittent bolus thermodilution cardiac output. Anesth Analg. 2012;115(1):82-7. 